\title{
Biochemical Evidence for Reduced Carnitine Palmitoyl Transferase 1 (CPT-1) Activity in Type 1 Diabetes Mellitus
}

\author{
Jill D. Jacobson ${ }^{1 *}$, L. Kurt Midyett ${ }^{2}$, Uttam Garg ${ }^{1,3}$, Ashley K. Sherman ${ }^{4}$ and Chetan Patel ${ }^{1,5}$
}

${ }^{1}$ Section of Endocrinology and Diabetes, Department of Pediatrics, Children's Mercy Hospitals and Clinics, University of Missouri-Kansas City School of Medicine, Kansas City, MO 64108, USA

${ }^{2}$ Division of Pediatric Endocrinology and Genetics, University of Kansas School of Medicine, Kansas City, KS, 66160, USA

${ }^{3}$ Department of Pathology and Laboratory Medicine, Children's Mercy Hospitals and Clinics, University of Missouri-Kansas City School of Medicine, Kansas City, MO 64108, USA

${ }^{4}$ Department of Medical Research, Children's Mercy Hospitals and Clinics, Kansas City, MO 6410, USA

5Section of Endocrinology, Department of Pediatrics, University of Arizona School of Medicine, Tucson, AZ 85721, USA

\begin{abstract}
Background: Type 1 diabetes mellitus is reportedly characterized by deficiencies of plasma total and free carnitine. Several inherited defects in fatty acid oxidation are associated with carnitine deficiency and with recurrent hypoglycemia. Hypoglycemia continues to be the major limiting factor in the management of type 1 diabetes. Herein, we sought to identify a subset of patients with abnormalities in carnitine profiles. Associations between carnitine abnormalities and glycemic control were also sought.
\end{abstract}

Methods: We performed a single center, prospective, observational study including 153 type 1 diabetic participants and 21 healthy control participants. We measured total, free, and acylcarnitine levels by high performance liquid chromatography-mass spectrometry. Glucose measurements were downloaded from patients' meters.

Results: In contrast to previous reports, no differences were found in free or total acylcarnitine levels between diabetic and control participants. Diabetic participants displayed high levels of C8 and C10 compared to controls. They also displayed biochemical evidence for reduced carnitine palmitoyl transferase 1 (CPT-1) enzymatic activity (as measured by free carnitine/C16 + C18) compared to controls. Patients with the lowest CPT-1 activity displayed a reduction in hemoglobin $A_{1 c}$ levels compared to the remaining population, but no unusual rates of hypoglycemia. We identified two patients who were heterozygous carriers of medium chain acyl-Co A dehydrogenase deficiency. These patients also displayed no detectable increased rates of hypoglycemia.

Conclusions: We conclude that deficiencies in free and total carnitine are not as common as previously reported in pediatric patients with type 1 diabetes. Patients with Type 1 diabetes display elevated medium chain acylcarnitines and biochemical evidence for a relative reduction in CPT-1 activity compared to the control population. We did not identify a subset of patients exhibiting both carnitine abnormalities and unusual rates of hypoglycemia.

Keywords: Carnitine; Hypoglycemia; Type 1 diabetes; Fatty acid oxidation; Carnitine palmitoyl transferase

\section{Background}

A small body of literature indicates that patients with type 1 diabetes display secondary carnitine deficiency. One study documented decreased free carnitine and increased acylcarnitine levels in children with type 1 diabetes in ketoacidosis, which normalized with insulin treatment and reversal of ketoacidosis [1]. Additional reports suggest that the prevalence of relative carnitine deficiency may be as high as thirty percent in diabetic children [2].

Approximately eleven disorders of fatty acid metabolism have been described, almost all of which are associated with abnormal carnitine or acylcarnitine levels and recurrent hypoglycemia [3]. The most common of these fatty acid oxidation disorders, medium chain acyl-CoA dehydrogenase deficiency (MCADD), classically presents as severe hypoglycemia during a prolonged fast or during acute illness. Patients with this disorder can remain completely asymptomatic for years [4].

Disorders of carnitine metabolism should be considered in the differential diagnosis of hypoglycemia [3,5,6]. In the setting of type 1 diabetes, however, the possibility of a fatty acid oxidation defect is generally not entertained. In diabetes, hypoglycemia is virtually always attributed to excessive insulinization. Meanwhile, hypoglycemia continues to be the major limiting factor in the management of individuals with type 1 diabetes [7,8]. Multicenter studies have demonstrated that intensive insulin therapy in diabetes leads to decreased long term morbidity and mortality. However, it is also associated with a two to three fold increase in severe hypoglycemia $[9,10]$

We hypothesized that a small subset of Type 1 diabetic subjects may have biochemical evidence for subclinical defects in fatty acid oxidation. We further speculated that the combination of heterozygous medium chain acyl CoA dehydrogenase deficiency (MCADD) with type 1 diabetes might result in a clinically significant reduction in carnitine levels.

*Corresponding author: Jill D. Jacobson, MD, Section of Endocrinology and Diabetes, Department of Pediatrics, Children's Mercy Hospitals and Clinics, University of Missouri-Kansas City School of Medicine, 2401 Gillham Road Kansas City, MO, USA, Tel:(816) 855-1979; Fax: (816) 855-1919; E-mail: jjacobson@cmh.edu

Received September 01, 2011; Accepted October 04, 2011; Published October 15, 2011

Citation: Jacobson JD, Midyett LK, Garg U, Sherman AK, Patel C (2011) Biochemical Evidence for Reduced Carnitine Palmitoyl Transferase 1 (CPT-1) Activity in Type 1 Diabetes Mellitus. J Diabetes Metab 2:144. doi:10.4172/2155 6156.1000144

Copyright: @ 2011 Jacobson JD, et al. This is an open-access article distributed under the terms of the Creative Commons Attribution License, which permits unrestricted use, distribution, and reproduction in any medium, provided the original author and source are credited. 
We hypothesized that we could identify a small subset of patients with both carnitine profile abnormalities and recurrent hypoglycemia. Such patients might be candidates for therapy using the safe and welltolerated oral carnitine supplements.

Herein, we performed carnitine profile analysis and correlated carnitine values with both hypoglycemic events and with $\mathrm{HbA}_{1 c}$.

\section{Methods}

\section{Study design}

This is a single center, prospective, observational study. All procedures and analyses were approved by the Children's Mercy Hospital Institutional Review Board and were in accordance with the Declaration of Helsinki.

\section{Participants}

The study group consisted of 153 Type 1 diabetic patients, ages 7 to 21 years, diagnosed on the bases of antibody status, absence of morbid obesity, and the absence of excessive insulin requirements. Inclusion criteria for diabetic patients included an 18 month duration of diabetes, requirement of insulin therapy, and documentation of at least 150 capillary glucose measurements over twelve weeks.

The mean age of diabetic patients was 12.9 years. The mean hemoglobin $\mathrm{A}_{1 \mathrm{c}}\left(\mathrm{HbA}_{1 \mathrm{c}}\right.$ ) was $8.7 \%$ (reference range $4.0 \%$ to $6.0 \%$ ). Treatment regimens included 1) multiple daily injections (MDI; $\mathrm{n}$ $=84) ; 2) \mathrm{NPH}$ and rapid insulin $(\mathrm{n}=9) ; 3)$ pump therapy $(\mathrm{n}=60)$. Exclusion criteria included: L-carnitine supplementation, steroid use, known seizure disorders, history of use of valproic acid or metformin, and impaired gastrointestinal or renal function.

The control group consisted of healthy volunteers $(n=14)$ and patients who had undergone thorough endocrine evaluations in our clinic which revealed no endocrine abnormalities $(n=7)$.

\section{Glucose measurements}

Hypoglycemic events were stratified into percentage of all capillary glucose measurements consistent with mild hypoglycemia ( $<3.3 \mathrm{mmol} / \mathrm{L})$, moderate hypoglycemia $(<2.75 \mathrm{mmol} / \mathrm{L})$, and severe hypoglycemia $(<2.2 \mathrm{mmol} / \mathrm{L})$. The mean and standard deviation of the glucose values were recorded.

\section{Carnitine measurements}

Heparinized blood was immediately spun at room temperature. Plasma was separated and frozen at $-20^{\circ} \mathrm{C}$ until shipment to Baylor University (Houston, Texas). Acylcarnitines were measured by Quattro Micro, LC-tandem mass spectrometer (Waters Corporation, Milford, MA). Free carnitine was quantitated, and samples underwent hydrolysis for total carnitine measurement. The interassay coefficient of variation for free, total, and individual acylcarnitines was less than $15 \%$.

\section{Genetic testing for MCADD}

Four patients with abnormally elevated acylcarnitines underwent genetic sequencing of the Acyl-Coenzyme A Dehydrogenase, Medium chain (ACADM) gene, encoding medium chain acyl-coA dehydrogenase. The coding exons and the flanking intronic sequences were PCR amplified and sequenced in forward and reverse directions, using automated fluorescent dideoxy sequencing methods and the GenBank (NCB) 10 NM_DODD16.2 as the reference sequence.

\section{CPT-1 enzymatic activity}

A recent study demonstrated that CPT-1 activity was assessed by the following published formula: free carnitine/(C16 + C18). We identified a subset of diabetic participants whose CPT-1 activity was greater than two standard deviations above the mean, based on data derived from our control population.

\section{Statistical analysis}

All data were analyzed using a general linear regression model and the means were compared using a simple t-test. Sigma Plot and SPSS were used in the analysis. The $95 \%$ confidence interval limits were identified.

\section{Results}

\section{Acylcarnitine levels}

Diabetic subjects displayed differences in several individual acylcarnitine values compared to healthy controls (Table 1). One difference was increased levels of the medium chain acylcarnitines, C8 and C10. In contrast to Type 2 diabetic patients, who are reported to display elevations in long chain acylcarnitines compared to controls [11], type 1 diabetic participants displayed significantly lower levels of most long chain acylcarnitines compared to controls (Table 1).

\section{CPT-1 activity}

CPT-1 deficiency is characterized by failure to form long chain acylcarnitines, resulting in high levels of long chain fatty acids and low levels of C16 and C18 acylcarnitines. We assessed CPT-1 enzymatic activity using the following published formula: free carnitine divided by (C16+ C18) [12]. This formula has been used in neonatal screening programs and has been shown to be more sensitive and specific for CPT-1 deficiency compared to either free carnitine alone or long chain acylcarnitines alone [12]. Patients with type 1 diabetes displayed statistically significant elevations of these ratios compared to controls, indicating a relative reduction in CPT-1 activity (Table 1).

\section{Total and free carnitine levels}

Free carnitine and total carnitine values did not differ between diabetic participants and control participants. Three diabetic subjects exhibited free carnitine values less than $14.1 \mu \mathrm{mol} / \mathrm{L}$ (two standard deviations below the mean of the control population). Their serum values are well within the range of that seen in research subjects treated with the carnitine-lowering pivalate-containing antibiotics [13]. Only one of these four patients displayed markedly low total serum carnitine levels $<10 \mu \mathrm{mol} / \mathrm{L}$, a level at which hypoglycemia has been described [14].

\section{Genetic testing}

Four patients exhibited medium chain acylcarnitine levels above the upper limits of normal for our lab, $>180 \mathrm{nmol} / \mathrm{L}$, similar to levels seen in heterozygous carriers of medium chain acyl-CoA dehydrogenase (MCAD) deficiency [15]. Genetic testing for the common mutations in the acyl-Coenzyme A dehydrogenase, C-4 to C-12 straight chain (ACDAM) gene was performed in all 4 individuals. Two patients exhibited the same common heterozygous missense mutation, c. $985 \mathrm{~A}>\mathrm{G}$ (p.K329E), upon sequencing of the entire coding exons of the ACADM gene. The c.985A $>$ G (p.K329E) is a common mutation found in patients with medium chain Acyl-CoA dehydrogenase deficiency. Thus, these two individuals were carriers of defects in the ACADM gene. The carrier frequency for ACADM mutations in North America 
Citation: Jacobson JD, Midyett LK, Garg U, Sherman AK, Patel C (2011) Biochemical Evidence for Reduced Carnitine Palmitoyl Transferase 1 (CPT1) Activity in Type 1 Diabetes Mellitus. J Diabetes Metab 2:144. doi:10.4172/2155-6156.1000144

Page 3 of 5

is approximately one in $70[15,16]$. Thus, our finding of 2 patients in our total population of 153 diabetic patients is concordant with the expected frequency for heterozygous mutations in the ADADM gene the general population. Although acylcarnitine levels were high, total and free carnitine levels were normal in these patients: free carnitine levels were 32.84 and $32.81 \mu \mathrm{mol} / \mathrm{L}$ and total carnitine levels were 36.48 and $35.78 \mu \mathrm{mol} / \mathrm{L}$ in the two individuals.

\section{Hypoglycemia in subsets of patients}

The thirty patients who displayed biochemical evidence for reduced CPT-1 activity displayed no increased rates of hypoglycemia. On the other hand, they did exhibit a significant reduction in $\mathrm{HbA}_{1 \mathrm{c}}$ levels (Table 2). No correlation was seen between duration of diabetes and measures of CPT-1 activity.

The three patients who displayed free or total carnitine levels more than 2 SD below the mean for the control population did not display an increased rate of hypoglycemia (Table 2). Even the one patient whose total carnitine values were even lower (below $10 \mu \mathrm{mol} / \mathrm{L}$ ) did not display unusual hypoglycemia (Table 2).
The two patients with documented mutations in ACDAM displayed very low percentages of hypoglycemia (Table 2).

\section{Carnitine levels and glycemic control in diabetic participants}

No general correlations were seen between free, total, or acylcarnitine levels and glycemic control as measured by hemoglobin $\mathrm{A}_{1 \mathrm{c}}$ or as measured by standard deviation of glucose measurements.

\section{Hemoglobin $\mathrm{A}_{1 \mathrm{c}}$ measurements}

The mean $\mathrm{HbA}_{1 \mathrm{c}}$ in our study population was $8.7 \%$. The mean $\mathrm{HbA}_{1 \mathrm{c}}$ for our entire population of diabetics at Children's Mercy Hospital is $9.19 \pm 3.01 \%(95 \% \mathrm{CI}=-0.739$ to $-0.161 \%$; $\mathrm{p}=0.002)$.

\section{Discussion}

We found biochemical evidence for a relative decrease in CPT1 enzymatic activity in a subset of patients with type 1 diabetes. The subset of patients displaying abnormally low CPT-1 activity exhibited significantly lower $\mathrm{HbA}_{1 \mathrm{c}}$ levels in the absence any detectable increased rates of hypoglycemia. This finding raises the question as to whether a reduction of CPT-1 activity may confer an advantage on glycemic

\begin{tabular}{|c|c|c|c|c|}
\hline & $\begin{array}{l}\text { Control Participants }(n=21) \\
\text { Mean } \pm \text { S.D. }\end{array}$ & $\begin{array}{l}\text { Diabetic Participants } \\
(n=153) \\
\text { Mean } \pm \text { S.D. }\end{array}$ & $\begin{array}{c}\mathbf{P} \\
\text { Value }\end{array}$ & $\begin{array}{l}95 \% \text { Confidence } \\
\text { Interval }\end{array}$ \\
\hline Age (years) & $13.20 \pm 3.17$ & $12.95 \pm 3.51$ & 0.757 & $(-1.35,1.85)$ \\
\hline Free Carnitine $(\mu \mathrm{mol} / \mathrm{L})$ & $28.92 \pm 7.42$ & $32.32 \pm 9.44$ & 0.115 & $(-7.64,0.84)$ \\
\hline Total Carnitine $(\mu \mathrm{mol} / \mathrm{L})$ & $33.78 \pm 7.76$ & $36.80 \pm 10.76$ & 0.216 & $(-7.82,1.78)$ \\
\hline Calculated acyl Carnitine ( $\mu \mathrm{mol} / \mathrm{L})$ (total-free) & $4.86 \pm 2.70$ & $4.48 \pm 3.48$ & 0.632 & $(-1.18,1.94)$ \\
\hline Calculated acyl:free ratio & $0.20 \pm 0.14$ & $0.15 \pm 0.14$ & 0.127 & $(-0.01,0.11)$ \\
\hline $\begin{array}{l}\text { Free carnitine/C16 + C18 } \\
\text { (CPT-1 activity) }\end{array}$ & $0.20 \pm 0.09$ & $0.28 \pm 0.11$ & $0.002^{*}$ & $(-0.13,-0.03)$ \\
\hline C3 (nmol/L) & $104.44 \pm 44.70$ & $127.44 \pm 61.18$ & 0.099 & $(-50.33,4.33)$ \\
\hline C3-DC (nmol/L) & $44.75 \pm 18.89$ & $37.16 \pm 19.61$ & 0.097 & $(-1.38,16.56)$ \\
\hline $\mathrm{C} 4(\mathrm{nmol} / \mathrm{L})$ & $121.64 \pm 45.85$ & $121.73 \pm 72.18$ & 0.996 & $(-32.08,31.90)$ \\
\hline C5 (nmol/L) & $185.26 \pm 55.99$ & $177.25 \pm 127.21$ & 0.777 & $(-47.62,63.64)$ \\
\hline C5-DC (nmol/L) & $31.63 \pm 12.40$ & $37.54 \pm 18.22$ & 0.152 & $(-14.01,2.19)$ \\
\hline C6(nmol/L) & $73.82 \pm 24.18$ & $65.42 \pm 32.23$ & 0.252 & $(-6.02,22.82)$ \\
\hline $\mathrm{C} 8(\mathrm{nmol} / \mathrm{L})$ & $62.45 \pm 20.57$ & $84.36 \pm 41.46$ & $0.019^{*}$ & $(-40.10,-3.72)$ \\
\hline C10(nmol/L) & $96.07 \pm 34.97$ & $130.04 \pm 65.89$ & $0.022^{*}$ & $(-62.94,-5.00)$ \\
\hline C10:1 (nmol/L) & $139.13 \pm 52.04$ & $134.05 \pm 51.84$ & 0.674 & $(-18.74,28.90)$ \\
\hline C12(nmol/L) & $75.09 \pm 18.07$ & $75.45 \pm 37.51$ & 0.966 & $(-16.80,16.08)$ \\
\hline C12:1 (nmol/L) & $90.82 \pm 30.45$ & $79.32 \pm 42.55$ & 0.233 & $(-7.48,30.48)$ \\
\hline C14(nmol/L) & $39.09 \pm 10.33$ & $38.22 \pm 24.05$ & 0.870 & $(-9.64,11.38)$ \\
\hline C14:1 (nmol/L) & $56.01 \pm 17.13$ & $69.23 \pm 38.11$ & 0.119 & $(-29.89,3.45)$ \\
\hline C16(nmol/L) & $79.29 \pm 15.05$ & $62.21 \pm 21.27$ & $<0.001^{*}$ & $(7.60,26.56)$ \\
\hline C16:1 (nmol/L) & $53.08 \pm 17.56$ & $46.61 \pm 22.08$ & 0.200 & $(-3.45,16.39)$ \\
\hline $\mathrm{C} 16-\mathrm{OH}(\mathrm{nmol} / \mathrm{L})$ & $17.87 \pm 4.21$ & $11.41 \pm 11.31$ & $0.011^{*}$ & $(1.53,11.39)$ \\
\hline C18(nmol/L) & $73.23 \pm 27.99$ & $64.37 \pm 25.96$ & 0.148 & $(-3.18,20.90)$ \\
\hline C18:1 (nmol/L) & $128.17 \pm 39.53$ & $130.81 \pm 53.07$ & 0.827 & $(-26.38,21.10)$ \\
\hline $\mathrm{C} 18-\mathrm{OH}(\mathrm{nmol} / \mathrm{L})$ & $13.67 \pm 6.70$ & $36.78 \pm 76.71$ & 0.171 & $(-56.25,10.03)$ \\
\hline $\mathrm{C} 18: 1-\mathrm{OH}(\mathrm{nmol} / \mathrm{L})$ & $17.39 \pm 6.64$ & $9.65 \pm 3.99$ & $<0.001^{*}$ & $(5.73,9.75)$ \\
\hline
\end{tabular}

*significantly different from controls

Table 1: Comparisons of control participants to diabetic participants. 


\begin{tabular}{|c|c|c|c|c|}
\hline Subsets of subjects & $\begin{array}{l}\% \text { glucoses } \\
<3.3 \mathrm{mmol} / \mathrm{L}\end{array}$ & $\%$ glucoses $<2.75 \mathrm{mmol} / \mathrm{L}$ & $\begin{array}{c}\% \text { glucoses } \\
<2.2 \mathrm{mmol} / \mathrm{L}\end{array}$ & $\mathrm{HbA}_{1 \mathrm{c}}$ \\
\hline All subjects $(n=153)$ & $6.5 \pm 5.7$ & $2.5 \pm 3.5$ & $0.5 \pm 1.4$ & $8.7 \pm 1.4$ \\
\hline Low CPT-1 subset $(n=30)$ & $6.4 \pm 3.9$ & $2.2 \pm 1.0$ & $0.4 \pm 0.1$ & $8.0 \pm 0.8$ * \\
\hline Normal CPT-1 subset $(n=123)$ & $6.5 \pm 6.0$ & $2.6 \pm 3.8$ & $0.5 \pm 1.4$ & $8.8 \pm 1.4$ \\
\hline ACDAM mutations $(n=2)$ & $4.00 \pm 2.8$ & 0.0 & 0.0 & $9.2 \pm 0.7$ \\
\hline Free carnitine greater than 2 SD below mean $(n=3)$ & $4.7 \pm 1.2$ & $1.7 \pm 1.2$ & 0.0 & $8.3 \pm 2.5$ \\
\hline Free carnitine less than $10 \mu \mathrm{mol} / \mathrm{L}(n=1)$ & 6.2 & 3.0 & 0.0 & 6.3 \\
\hline
\end{tabular}

${ }^{*}$ significantly lower than those with normal CPT-1 levels; $p=0.0003 ; 95 \% \mathrm{Cl}(-1.22,-0.27)$

Table 2: Comparisons of subsets of subjects with altered carnitine measurements.

control in type 1 diabetes. In fact, pharmacologic inhibition of CPT-1 activity has been clinically tested in the past in human patients with type 2 diabetes. These inhibitors were shown to be effective in decreasing hepatic glucose production and increasing glucose utilization $[9,17,18]$.

The mechanisms for reduced CPT-1 activity in the diabetic population are not clear. Both hyperglycemia and hyperinsulinism have been shown to be associated with reduced CPT-1 activity [19]. A recent study reports suggest that recurrent hypoglycemia can inhibit CPT-1 gene expression in the hypothalamus in mice [20]. Perhaps fluctuations of glucose in type 1 diabetes lead to alterations in CPT-1 activity over time. However, in the current study, no correlation was seen between duration of diabetes and measures of CPT-1 activity.

A limitation of our study was the measurement of intermittent glucoses. Continuous glucose monitoring systems would have had the advantage of detecting nocturnal or episodic hypoglycemia in this study.

We found no differences in total or free carnitine levels in our patient population compared to healthy non-diabetic controls. This finding differs from previous reports showing lower levels of total and free carnitine in diabetic participants compared to healthy controls [21-23]. It is possible that contemporary therapy of diabetes is less likely to be associated with carnitine deficiency compared to historic regimens. In our study as well as in others', no correlations were found between total or free carnitine levels and glycemic control, as measured by $\mathrm{HbA}_{1 \mathrm{c}}$ values and glucose standard deviation scores [2,21].

We detected elevated levels of C8 and C10 acylcarnitines. These findings are reminiscent of findings in a recent study of type 2 diabetic subjects who displayed elevated levels of C6, C8 and summed C10 to C14 acylcarnitine levels [11]. In contrast, we detected low levels of the long chain acylcarnitines $\mathrm{C} 16, \mathrm{C} 16-\mathrm{OH}$, and $\mathrm{C} 18: 1-\mathrm{OH}$, which can be seen with impaired formation of long chain acylcarnitines, as occurs with impairment of CPT-1 deficiency.

We identified two patients with heterozygous ACDAM mutations and three patients with abnormally low levels of free carnitine and one patient who exhibited total and free carnitine levels below $10 \mu \mathrm{mol} / \mathrm{L}$. None of these subsets of participants displayed increased rates of hypoglycemia. The degree of carnitine deficiency that can be associated with hypoglycemia is controversial. Experts in hypoglycemia have suggested that tissue concentrations of free carnitine probably have to be less that $10 \%$ of normal before hypoglycemia occurs [3]. However, this speculation is based largely on studies in patients with very low levels of carnitine associated with carnitine transporter defects associated with mutations of the OCTN2 gene, [24]. In these patients, L-carnitine therapy improves survival, even though tissue levels of carnitine remain $<10 \%$ of normal $[3,25]$.

Suppression of CPT-1 activity would be expected to be associated with reduced long chain acylcarnitines, but increased levels of long chain free fatty acids. Further analysis of free fatty acids in subgroups of patients with suppressed CPT- 1 activity may be in order.

In conclusion, carnitine deficiency appears to be less common in our pediatric population of type 1 diabetes compared to historical reports. The main abnormalities noted in carnitine metabolism in our diabetic population were a biochemical evidence for impairment in CPT-1 activity and elevations in medium chain acylcarnitines. No increased percentages of hypoglycemic events were seen, even in patients with the most extreme carnitine abnormalities. A significant reduction in $\mathrm{HbA}_{1 \mathrm{c}}$ was seen in patients with impaired CPT-1 activity compared to the remaining population.

\section{Acknowledgments}

This work was supported by a grant from the Katherine B. Richardson Foundation administered by the Children's Mercy Hospital

\section{Conflicts of Interest}

The authors have no disclosures related to this work.

\section{References}

1. Soltesz G, Melegh B, Sandor A (1983) The relationship between carnitine and ketone body levels in diabetic children. Acta Pediatr Scand 72: 511-515.

2. Winter SC, Simon M, Zorn EM, Szabo-Aczel S, Vance WH, et al. (1989) Relative carnitine insufficiency in children with type I diabetes mellitus. Am J Dis Child 143: 1337-1339.

3. Stanley CA (2004) Carnitine deficiency disorders in children. Ann N Y Acad Sc 1033: $42-51$.

4. Schatz UA, Ensenauer R (2010) The clinical manifestation of MCAD deficiency: challenges towards adulthood in the screened population. J Inherit Metab Dis 33: $513-520$

5. Hsu HW, Zytkovicz TH, Comeau AM, Strauss AW, Marsden D, et al. (2008) Spectrum of medium-chain acyl-CoA dehydrogenase deficiency detected by newborn screening. Pediatrics 121: 1108-1114.

6. Kuwajima M, Fujihara H, Sei H, Umehara A, Sei M, et al. (2007) Reduced carnitine level causes death from hypoglycemia: possible involvement of suppression of hypothalamic orexin expression during weaning period. Endocr J 54: 911-925.

7. Cryer PE (2008) The barrier of hypoglycemia in diabetes. Diabetes 57: 3169 3176. 
Citation: Jacobson JD, Midyett LK, Garg U, Sherman AK, Patel C (2011) Biochemical Evidence for Reduced Carnitine Palmitoyl Transferase 1 (CPT1) Activity in Type 1 Diabetes Mellitus. J Diabetes Metab 2:144. doi:10.4172/2155-6156.1000144

Page 5 of 5

8. Heller SR (2008) Minimizing hypoglycemia while maintaining glycemic control in diabetes. Diabetes 57: 3177-3183.

9. DCCT Research Group T (1991) Epidemiology of severe hypoglycemia in the diabetes control and complications trial. The DCCT Research Group. Am J Med 90: 450-459

10. DCCT Research Group T (1995) Adverse events and their association with treatment regimens in the diabetes control and complications trial. Diabetes Care 18: 1415-1427

11. Adams SH, Hoppel CL, Lok KH, Zhao L, Wong SW, et al. (2009) Plasma acylcarnitine profiles suggest incomplete long-chain fatty acid beta-oxidation and altered tricarboxylic acid cycle activity in type 2 diabetic African-American women. J Nutr 139: 1073-1081.

12. Fingerhut R, Roschinger W, Muntau AC, Dame T, Kreischer J, et al. (2001) Hepatic carnitine palmitoyltransferase I deficiency: acylcarnitine profiles in blood spots are highly specific. Clin Chem 47: 1763-1768.

13. Abrahamsson K, Eriksson BO, Holme E, Jodal U, Lindstedt S, et al. (1994) Impaired ketogenesis in carnitine depletion caused by short-term administration of pivalic acid prodrug. Biochem Med Metab Biol 52: 18-21.

14. Abrahamsson K, Holme E, Jodal U, Lindstedt S, Nordin I (1995) Effect of short-term treatment with pivalic acid containing antibiotics on serum carnitine concentration--a risk irrespective of age. Biochem Mol Med 55: 77-79.

15. Blois B, Riddell C, Dooley K, Dyack S (2005) Newborns with C8-acylcarnitine level over the 90th centile have an increased frequency of the common MCAD 985A>G mutation. J Inherit Metab Dis 28: 551-556.

16. Giroux S, Dube-Linteau A, Cardinal G, Labelle Y, Laflamme N, et al. (2007) Assessment of the prevalence of the 985A>G MCAD mutation in the FrenchCanadian population using allele-specific PCR. Clin Genet 71: 569-575.

17. Hubinger A, Knode O, Susanto F, Reinauer H, Gries FA (1997) Effects of the carnitine-acyltransferase inhibitor etomoxir on insulin sensitivity, energy expenditure and substrate oxidation in NIDDM. Horm Metab Res 29: 436-439.

18. Ratheiser K, Schneeweiss B, WaldhausI W, Fasching P, Korn A, et al. (1991) Inhibition by etomoxir of carnitine palmitoyltransferase I reduces hepatic glucose production and plasma lipids in non-insulin-dependent diabetes mellitus. Metabolism 40: 1185-1190.

19. Rasmussen BB, Holmback UC, Volpi E, Morio-Liondore B, Paddon-Jones D, et al. (2002) Malonyl coenzyme A and the regulation of functional carnitine palmitoyltransferase-1 activity and fat oxidation in human skeletal muscle. $J$ Clin Invest 110: 1687-1693.

20. Poplawski MM, Mastaitis JW, Mobbs CV (2011) Naloxone, but not valsartan, preserves responses to hypoglycemia after antecedent hypoglycemia: role of metabolic reprogramming in counterregulatory failure. Diabetes 60: 39-46.

21. Mamoulakis D, Galanakis E, Dionyssopoulou E, Evangeliou A, Sbyrakis S (2004) Carnitine deficiency in children and adolescents with type 1 diabetes. J Diabetes Complications 18: 271-274.

22. Cederblad G, Lundholm K, Schersten T (1977) Carnitine concentration in skeletal muscle tissue from patients with diabetes mellitus. Acta Med Scand 202: 305-306.

23. H, Evangeliou A, Bervoets K, Eckert I, Sewell A (1994) Carnitine esters in metabolic disease. Eur J Pediatr 153: 57-61.

24. Wang Y, Ye J, Ganapathy V, Longo N (1999) Mutations in the organic cation/ carnitine transporter OCTN2 in primary carnitine deficiency. Proc Natl Acad Sc U S A 96: 2356-2360.

25. Stanley CA, DeLeeuw S, Coates PM, Vianey-Liaud C, Divry P, et al. (1991) Chronic cardiomyopathy and weakness or acute coma in children with a defect in carnitine uptake. Ann Neurol 30: 709-716 